\title{
Miscel-lània
}

\author{
BEATRIZ RÓDENAS CEREZO1
}

\section{«Aguantar...» Agencia cultural híbrida en los conflictos de género dentro de los matrimonios transnacionales africanos}

\author{
«Aguantar...» Hibrid Cultural Agency in Gender Conflicts \\ within African Transnational Marriages
}

\section{RESUMEN}

El paradigma teórico hegemónico que domina los estudios sobre violencia de género establece que la causa última de esta violencia descansa en la desigualdad de relaciones de poder entre mujeres y hombres (Red2Red, 2011). Sin negar una concepción estructural de la violencia de género, el presente artículo pretende abordar el caso de mujeres transmigrantes procedentes de África subsahariana en espacios rurales de Aragón, con el objetivo de demostrar cómo los feminismos afrocéntricos pueden visibilizar otro tipo de variables para explicar la violencia de género en la pareja (IPV). A través del trabajo de campo etnográfico realizado en esta zona, se intenta integrar la perspectiva estructural de la violencia de género con la agencia, dando como resultado procesos de hibridación donde la figura emergente de «una mujer con experiencia» se convierte en posible estrategia de resolución de la violencia, un proceso que ejemplifica la agencia cultural híbrida de las sociedades africanas.

Palabras clave: violencia de género en la pareja (IPV), migración africana, feminismos africanos/afrocéntricos, agencia cultural híbrida.

\section{Abstract}

The hegemonic theoretical paradigm that dominates studies on gender violence establishes that the ultimate cause of this violence rest on inequality in power relations between women and men (Red2Red, 2011). Without denying a structural conception of gender violence, this article aims to address the case of transmigrants from sub-Saharan Africa in rural areas of Aragon, with the aim of demonstrating how Afrocentric feminisms can make visible other types of variables to explain of intimate partner violence (IPV). Through ethnographic fieldwork carried out in this area, we try to integrate the structural perspective of gender violence with the agency, resulting in hybridization processes where the emerging figure of «an experienced woman» becomes a possible strategy for the resolution of violence, a process that exemplifies the hybrid cultural agency of African societies.

Keywords: Intimate Partner Violence (IPV), African migration, African / Afrocentric feminisms, hybrid cultural agency. 


\section{SUMARIO}

1.- Presentación: ubicando la investigación. 2.- Planteamientos teóricos: desde los procesos de hibridación a los feminismos africanos/afrocéntricos; una lectura particular de la violencia de género en la pareja (IPV). 3.- Apuntes metodológicos. 4.- Narraciones etnográficas: conflictos y estrategias de resolución en familias transnacionales africanas. 5.- Análisis afrocéntricos de la violencia de género y agencia cultural híbrida en contexto migratorio: el caso de «una mujer con experiencia». - Referencias bibliográficas.

\section{Presentación: ubicando la investigación}

- ¿Qué podemos hacer por nuestra amiga si creemos que está pasando por «eso»?

(Polifonía de voces) - ... hay que hablar... que aguante...
(Mujer divorciada Mali1)- no, no hay que aguantar...
(Mujer divorciada Mali2)- si le dices que no aguante, y luego se divorcia, la culpa es para ti...
(Mujer casada Gambia 1) - todo no se puede aguantar...
Grupo de discusión (2018)

Problemas dentro del matrimonio existen en todas las sociedades, independientemente de la latitud en la que nos encontremos. En Occidente se ha teorizado desde el feminismo una esfera doméstica, separada de la pública, y carente de poder político y económico, donde se ubica a las mujeres, a la vez que se conceptualiza este escenario como la fuente de la subordinación de la mujer frente a los hombres (Rosaldo, 1979; 1980). Y en este planteamiento, los conflictos matrimoniales se analizan en términos de relaciones de género, unas relaciones poco horizontales y donde las mujeres están en franca desventaja para reivindicar sus derechos. Partiendo de este escenario, la producción teórica se ha ido desarrollando alrededor de la violencia de género, en este caso que planteamos, en la pareja, producción teórica ubicada en un paradigma teórico, con unas herramientas metodológicas, y con unas preguntas teóricas concretas:

El esfuerzo de iluminar una zona oscura de la vida social, la fuerza del foco y sus límites ha creado un objeto específico: la violencia de género, fruto de la desigualdad de poder, estructural aunque se manifieste individualmente, y socialmente transversal, es decir que puede afectar a todas las mujeres (Red2Red, 2011: 14-15).

El paradigma teórico hegemónico que domina este tipo de estudios toma: «cautela por plantear posibles relaciones entre variables, que pueden enturbiar el hecho claro e inexcusable según el cual, la causa última de la violencia de género descansa en la desigualdad en las relaciones de poder entre mujeres y hombres» (Red2Red, 2011: 14-15). Sin embargo existen estudios que plantean la necesidad de centrar las investigaciones en, por ejemplo, «factores de riesgo», sin negar una concepción estructural de la violencia de género:

El hecho de que, desde posturas feministas, se siga rechazando cualquier explicación más allá de la que remite a la dominación masculina —aunque puede 
ser hasta útil para la causa de todas aquellas mujeres que no acumulan más opresiones que la de género (es el caso de mujeres blancas, autóctonas, de clase media-alta, con elevado nivel formativo, etc.) - es claramente contraproducente para aquellas que ocupan posiciones más precarizadas, y esto no es algo que, desde posiciones progresistas, se pueda defender (Damonti, 2019: 514).

En este mismo sentido, otras autoras también han destacado la importancia de los análisis interseccionales para ubicar a las diferentes mujeres en sus propios contextos de violencia de género. Crenshaw (2012) ha puesto de manifiesto que cuando el feminismo no se pregunta por las implicaciones raciales, reproduce la subordinación de las mujeres de color:

El problema no es simplemente que las mujeres que dominan el movimiento contra la violencia sean diferentes a las mujeres de color, sino que a menudo tienen el poder para determinar, o bien a través de recursos materiales o retóricos, si las diferencias interseccionales de las mujeres de color se incorporan o no en la formulación de una política determinada. (Crenshaw, 2012: 114).

Lo que pretendemos en el presente artículo es demostrar de qué manera los procesos de hibridación han brindado a las mujeres transmigrantes africanas una herramienta para lidiar con los conflictos de género en la pareja. Partiendo de su propio bagaje cultural y de sus experiencias sobre cómo funcionan las relaciones entre marido y mujer en África, han desarrollado sus propias estrategias para enfrentarse a las ideologías de género predominantes en las sociedades de destino. Para ello focalizaremos el análisis en el caso de las mujeres migrantes subsaharianas, procedentes de Mali, Gambia y Senegal, cuyos contextos de destino se ubican en espacios rurales de la Comunidad de Aragón, representado por la ficcionada comarca de La Aldahuela de Osca ${ }^{2}$ como comarca representativa de estos patrones migratorios. La ruta seguida por estas mujeres como esposas reagrupadas las ha llevado a definirlas, en muchas ocasiones, como anexos a los proyectos migratorios de sus esposos, obviando que una vez en destino, logran desarrollar en la mayoría de los casos, su propio proyecto migratorio (Azoulay y Quiminal, 2002; Jabardo, 2005; Konaté, 2010; Ródenas, 2014). El contexto migratorio transnacional en el que se mueven, donde cultivan sus relaciones familiares extensas transnacionales entre África subsahariana y España, definen a la familia transnacional como: «families that live some or most of the time separated from each other, yet hold together and create something that can be seen as a feeling of collective welfare and unity, namely «familyhood», even across national borders» (Bryceson y Vuorela, 2002: 15). Este espacio transnacional se convierte en un contexto fértil donde generar prácticas híbridas, a la vez que supone, como veremos en los próximos epígrafes, un proceso migratorio de estrés que puede llegar a explicar (pero no a justificar) algunos de los factores de riesgo que originarían las situaciones de violencia en la pareja.

2 Se ha procedido a mantener el anonimato en todo lo posible, para proteger a las informantes implicadas, así como proteger el trabajo imprescindible que realizan mis compañeras. Aprovecho para agradecer a mis compañeras de equipo y a las participantes en la investigación el haber hecho posible la existencia de este trabajo; así como las lecturas que de él hicieron evaluadores externos. 
Acercarnos a estos debates implica repasar, primero, cómo conceptualizar los procesos de hibridación en contexto africano para, a continuación, revisar las investigaciones en el ámbito africano sobre violencia de género en la pareja (Intimate Partner Violence: IPV, por sus siglas en inglés). Por último, utilizaremos lo que, en los últimos años, se ha ido denominando «feminismos africanos y/o afrocéntricos», en particular algunas lecturas que autoras africanas han hecho del género y de las relaciones de género, para intentar ubicar las estrategias híbridas desarrolladas por estas mujeres, en su propio contexto.

\section{Planteamientos teóricos: desde los procesos de hibridación a los feminismos africanos/afrocéntricos; una lectura particular de la violencia de género en la pa- reja (IPV)}

Los procesos de hibridación están estrechamente relacionados con los propios procesos de posmodernidad y glocalización que actualmente dominan el panorama presente (Rodríguez-García, 2013). En una obra ya clásica, García Canclini (1990) planteó el largo recorrido que la hibridez ha tenido en las culturas latinoamericanas. En un contexto de «densa interculturalidad» el incremento de los procesos de hibridación daba como resultado: «ciertas relocalizaciones territoriales relativas, parciales, de las viejas y nuevas producciones simbólicas» (García Canclini, 1990: 228). De esta forma, García Canclini utilizaba la producción cultural para analizar los intercambios, las identidades de fronteras y los procesos híbridos, para explicar las continuas readaptaciones entre lo moderno y lo tradicional en el mundo latinoamericano. En una obra posterior, con el objetivo de contestar a las críticas que se le hicieron de imprecisión terminológica, García Canclini definió los procesos de hibridez como: «procesos socioculturales en los que estructuras o prácticas discretas, que existían en forma separada, se combinan para generar nuevas estructuras, objetos y prácticas» (García Canclini, 1999). La obra de García Canclini está repleta de ejemplos de estos procesos de hibridación:

La misma combinación de prácticas científicas y tradicionales -ir al médico y al curandero- es una manera transaccional de aprovechar los recursos de ambas medicinas, con lo cual los usuarios revelan una concepción más flexible que el sistema médico moderno sectarizado en la alopatía, y que muchos folcloristas o antropólogos que idealizan la autonomía de las prácticas tradicionales. Desde la perspectiva de los usuarios, ambas modalidades terapéuticas son complementarias, funcionan como repertorios de recursos a partir de los cuales efectúan transacciones entre el saber hegemónico y el popular (García Canclini, 1990: 325).

De la misma forma, se han puesto de manifiesto cómo estos procesos de hibridación se han desarrollado también en contexto africano. Iniesta (2010) ha subrayado que: «el pensamiento integrador de la tradición africana favorece la incorporación de elementos ideológicos externos, subordinándolos y articulándolos en su estructura, abierta a la multiplicidad» (Iniesta, 2010: 138). En el caso africano, la mayoría 
de los procesos híbridos se han enmarcado en contextos de resistencia y agencia, en la misma línea de teorización que Bhabha (2002) usó las prácticas de hibridación para analizar los espacios de emancipación y de resistencia a la colonización en el sureste asiático. En palabras de Appert (2016):

Through practices of syncretism and hybridity, emergent African popular cultures mediated between the colonially imposed binaries of space, place, and time that, whether «invented» or «real», were clearly central to local understandings of the displacements of colonialism and in particular, new forms of urban existence in Africa (Appert, 2016: 281).

De manera similar, Bakare-Yusuf (2011) ha explicado cómo se ubica el pensamiento local africano en lo plural, huyendo de las trampas de la pureza, la autenticidad y el esencialismo. Ella se focaliza en el caso Yoruba, y expone que la estructura profunda de la sociedad nigeriana es politeísta y polirrítmica, asemejándose a las estructuras interrelacionales y múltiples que los teóricos posmodernistas han descrito. En palabras de Bakare-Yusuf:

En este punto de vista, la cultura Yoruba ocupa un espacio impuro y bastardo, que invita a la mezcla y al diálogo productivo con su exterior. Es este tipo de conocimientos locales destrozados mezclados que encuentro productivos: donde los platos sobresalgan de casas de barro, Nike se pasea en la selva y pegatinas de Mercedes se mezclan con fetiches locales en la cabina del conductor de autobús. En miles de ejemplos de este tipo, la cultura Yoruba muestra sus poderes polirrítmicos de acomodación, adaptándose y apropiándose de influencias externas, así como transformando internamente las propias [...] El indigenismo, si es que existe, existe en una forma de yuxtaposiciones culturales que están perfectamente vinculadas a través de modos confiables de la agencia cultural. (Bakare-Yusuf, 2011: 46).

Y es justo esta idea de agencia cultural híbrida, desde donde vamos a plantear cómo las mujeres migrantes africanas han desarrollado sus propias estrategias (diferentes a las de la sociedad de acogida) para enfrentarse a las situaciones conflictivas y violentas en el seno de los matrimonios transnacionales. Partiendo de un bagaje africano polirrítmico, tendiente al sincretismo y la multiplicidad, y habiendo recorrido un proceso migratorio que las ubica en contexto transnacional y de «densa interculturalidad», se encuentran en una posición ideal para desarrollar esas prácticas de agencia cultural híbrida que utilizan para enfrentarse a la violencia en la pareja, y que pretendemos visibilizar en el presente estudio. Pero para poder hacerlo, recorreremos primero los principales senderos teóricos que han analizado la violencia de género en contexto africano.

Para abordar este cometido, podemos rescatar las principales teorías que aparecen en la literatura afrocéntrica sobre violencia doméstica, a partir de las diferentes investigaciones sobre la violencia de género en la pareja (IPV). Bowman (2003) recoge cinco categorías generales para enmarcar las diferentes teorías sobre las causas de la violencia de género, usadas para África, y subraya una última (sexta) categoría infrautilizada en el contexto africano: 
a) teorías basadas en derechos humanos individuales, orientadas por nociones de autonomía que entran en conflicto con la experiencia más relacional de las mujeres en general, y con las sociedades africanas en particular, donde no se suele priorizar lo individual

b) explicaciones feministas, que subrayan las desigualdades de género en África, y enmarcan la lucha contra la violencia de género en una lucha más general por la igualdad de género

c) explicaciones culturales, que enfatizan la cultura tradicional africana y la conformidad hacia los roles tradicionales de género, enfocando temas como la poligamia, el precio de la novia, matrimonios tradicionales, el poder de la familia extensa, las obligaciones económicas tradicionales... etc., sin tener en cuenta que la cultura en África varía entre regiones, grupos, etc., cambia históricamente, y es contestada dentro del propio grupo

d) explicaciones basadas en el cambio social y por sociedades en transición: el cambio social experimentado actualmente, intensificado debido a la modernización, urbanización, migración... etc., provoca el fallo del control del comportamiento de los hombres por parte de las normas tradicionales

e) cultura de la violencia, relacionada con la herencia colonial, las guerras civiles... etc.

f) y, significativamente, ausencia de explicaciones psicológicas y económicas; sin embargo son explicaciones usadas muy habitualmente para los casos de violencia de género que ocurren en Estados Unidos de América: «the psychology versus culture dichotomy recapitulates the traditional, and racist, stereotype that associates the West with reason and depicts non-Western people as driven by irrational forces» (Bowman, 2003: 858).

De la misma manera, resulta muy revelador cómo otras autoras han incidido también en este uso diferenciado de explicaciones para la violencia de género, según sea la procedencia de las mujeres implicadas:

But when do certain acts become «culture»? Spousal murders (by shooting, stabbing, lethal injection, running over by car, etc.) are rampant in the United States and are often described by Americans as «crimes of passion». More women are raped in the United States than in most African countries, but Americans describe the problem as «violence against women», not «culture» (Nnaemeka, 2003: 372).

A continuación, partiendo de estas líneas teóricas previas, vamos a ubicar este trabajo en un contexto teórico que intente relacionar las explicaciones referentes a la estructura social, con la acción/agencia híbrida de los individuos implicados. En el caso que pretendemos etnografiar, partimos del proceso migratorio como un proceso de transición, de cambio, especialmente conflictivo, donde diferentes factores de estrés entran en juego. De este modo, a lo largo de los siguientes párrafos intentaremos ilustrar de qué manera se ejerce agencia, es decir, qué estrategias se pueden utilizar, en un contexto de cambio transnacional donde se producen conflictos en la pareja.

Tomando como punto de partida algunas de las posiciones que AkinsulureSmith et al. (2013) han planteado, podemos señalar que existen varios estudios que 
sugieren que las experiencias migratorias actúan como inductores de cambio que pueden convertirse en una fuente de conflicto familiar (Akinsulure-Smith et al., 2013; Pan et al., 2006). Sin embargo, quisiéramos alejarnos muy conscientemente de la explicación que subyace a muchos de los trabajos que utilizan esta argumentación: no quisiéramos transmitir la idea de que mujeres procedentes de culturas con sistemas de género menos igualitarios, al entrar en contacto con sociedades de destino donde las mujeres están inmersas en sistemas de género más igualitarios, toman conciencia de su sumisión, y surgen los problemas, debido a que las mujeres inmigrantes quieren asumir esos roles de género comparativamente más igualitarios. Esta premisa resulta más o menos explícita en muchos estudios sobre género, migración y, particularmente, África: «As immigrants become more acculturated, they may alter their ideologies to accommodate the presence of comparatively more egalitarian U.S. gender roles» (Raj y Silverman, 2002: 370). Otros ejemplos:

Yet due to the fact that historically, within African cultures, there has been no acknowledgement of women's individual human rights and rights to equality within male/female relationships [...] In spite of being educated in the United States or becoming acculturated, these husbands did not become more accepting of gender equality or women's rights (Ting y Panchanadeswaran, 2009: 831-834).

Este tipo de explicaciones ignora tanto la construcción intelectual y política de lo que se han llamado otros feminismos desde una perspectiva interseccional (feminismos negros, africanos, musulmanes, del tercer mundo, etc.) como la propia crítica interna que los feminismos hegemónicos de «Occidente» han desarrollado, pero que también han descuidado en muchas ocasiones (Abu-Lughod, 2002; Amos y Parmar, 1984; Carby, 2012; Mohanty, 2008). En este sentido, existe toda una serie de trabajos de recopilación y traducción que intentan dar forma a estos dos objetivos: tanto a una deconstrucción del feminismo hegemónico, como a una construcción de otras formas de feminismos (Jabardo, 2012; Suárez Navaz y Hernández, 2008; vv.aa, 2004).

Sin negar los constantes e intensos procesos de cambio que experimentan todas las sociedades (incluidas las africanas), la experiencia migratoria supone una variedad de contradicciones y tensiones que en ocasiones deriva en cuestionamientos de algunos de los planteamientos de origen: «c'est la rencontre entre deux modes de contrôle sur les femmes qui aiguise et multiplie les tensions entre les hommes et les femmes» (Azoulay y Quiminal, 2002:88). En el caso que nos ocupa, las familias de origen subsahariano (Mali, Gambia, Senegal) que han llegado a destino (Aragón rural), adaptan sus estrategias al nuevo contexto transnacional, asumiendo nuevas responsabilidades y roles. En esa situación, muchas mujeres han hecho constar cómo no sólo la violencia física o psicológica aparece, sino que surgen narraciones de conflictos centrados en intereses económicos que la literatura sobre violencia de género ha ido enmarcando en lo que se conoce como violencia económica: «hey cited experiences of financial and emotional manipulation, physical abuse, being silenced during family discussions, verbal abuse and bullying. The women stated that their husbands were in control of the family's finances and controlled all other aspects of their lives» (Ogunsiji et al, 2011:1662). Rey Anacona (2013) ha puesto de 
manifiesto como existen dos tipos de violencia de pareja que no se han estudiado suficientemente, la violencia económica y la negligencia o descuido:

Maltrato económico. Forzar a la otra persona a depender económicamente del agresor, no dejándola trabajar o por otros medios; ejercer control sobre los recursos financieros de la víctima o explotarla económicamente. Negligencia. No proporcionar (o no hacerlo debidamente) recursos financieros o materiales, información o servicios a la pareja, a pesar de que el agresor está obligado legalmente a hacerlo; no brindar ayuda económica o material a la pareja cuando ésta lo necesitaba y el victimario estaba en capacidad de hacerlo o no advertir a la pareja sobre algún peligro para su integridad física o psicológica (Rey Anacona, 2013: 146).

Es justamente en esta línea teórica donde podemos enmarcar las experiencias recogidas en la investigación que presentamos. Como veremos en los próximos párrafos, las narraciones de las mujeres que han participado en la investigación giran en torno a este tipo de conflictos, como formas de tensión en los matrimonios. Además no hemos de olvidar que, entre la literatura afrocéntrica dedicada al IPV, una de las hipótesis de partida con las que se cuenta es cómo la estructura social, económica y jurídica de destino pone en una situación delicada a los inmigrantes hombres, lo que actúa como un factor de estrés más que influye en el posible desencadenamiento de los conflictos maritales (Akinsulure-Smith et al., 2013; Bowman, 2003; Ogunsiji et al., 2011; Pan et al., 2006): «many male participants viewed their economic stressors as the root cause of IPV» (Akinsulure-Smith et al., 2013: 6); «men report feelings of helplessness and uselessness because they have lost their role as the breadwinner and many try to regain a sense of control through violence» (Pan et al., 2006: 39).

En un intento de integrar esta perspectiva estructural con la agencia de los individuos, nuestro siguiente objetivo es centrarnos en las posibilidades y estrategias de las mujeres migrantes africanas para enfrentarse a este tipo de situaciones. Y es en este intento de comprender su agencia cuando es necesario traer a colación las lecturas que algunas autoras africanas han hecho de las relaciones de género. Son varios los estudios que recogen una forma particular de proceder de las mujeres africanas que usa las redes que proporcionan la comunidad o la familia extensa, para resolver los conflictos en la pareja. Amadiume (2018) ya puso de manifiesto en su análisis de la sociedad igbo de Nnobi (Nigeria) en 1987, cómo las coesposas del linaje trataban los conflictos maritales: si encontraban que el culpable era el marido, lo sancionaban a través del cabeza de linaje, o lo remitían a sus hermanas (Amadiume, 2018: 121). De este modo, para las mujeres, lo importante era el apoyo de sus hermanos, su propio patrilinaje no renunciaba al derecho a protegerlas (Amadiume, 2018: 132). De esta manera, en cualquier caso de violencia que surgiera, los parientes estaban cerca para defender a su hermana, hija o madre (Amadiume, 2018: 190).

En el contexto migratorio contemporáneo, también se han encontrado evidencias de este tipo de intervenciones de la familia extensa y/o la comunidad para 
proteger a las mujeres: «the women were accustomed to the informal network of extended family members as mediators in situations of IPV in Africa, and the absence of these extended family members in Australia created a vacuum» (Ogunsiji et al., 2011: 1661). Akinsulure-Smith y equipo (2013) encontraron que entre los inmigrantes de África Occidental en Estados Unidos, el primer paso ante una situación de violencia de pareja (IPV), es acudir a la familia; a continuación, acudir a los mayores, o a los líderes religiosos, referentes de la comunidad social/cultural; y, por último, utilizar los recursos fuera de la comunidad, disponibles en las sociedades de destino: "conflicts are resolved through networks led by immediate and extended family, community elders, and religious and spiritual leaders» (Akinsulure et al., 2013:10). El mismo mecanismo ha sido reportado entre los refugiados somalíes en Estados Unidos: «in addition to Islamic traditions, family members and community elders are frequently identified as a resource for resolving conflict between spouses. Traditionally, the wife would consult with the men in her family and then the men would talk to the husband» (Pan et al., 2006: 39).

Sin perder de vista esta fuente de recursos de la que disponen las protagonistas, es necesario subrayar cómo en ocasiones la comunidad puede convertirse también en una barrera para escapar de los conflictos maritales, debido a la presión ejercida por la familia extensa y por la comunidad para mantener el matrimonio (Sokoloff, 2008; Ting y Panchanadeswaran, 2009), y/o por el aislamiento que puede sufrir la mujer como consecuencia de acudir a los recursos sociales externos a la comunidad que se disponen en las sociedades de destino (Akinsulure-Smith et al., 2013; Ogunsiji et al., 2011; Ródenas, 2014; Ting y Panchanadeswaran, 2009):

There are legal tensions between protecting the mother from the husband and, in the process, cutting her off from the community [...] due to limited social networks, such an order of protection might mean that the woman cannot interact freely in the community without coming into contact with her abusive spouse [...] Those women who did contact police paid a high price: isolation and loss of extended family networks and community (Akinsulure-Smith et al., 2013: 9).

Para captar en su totalidad estos procesos, es necesario comprender cómo funcionan las relaciones de género en África, y porqué los feminismos africanos han incluido nociones de complementariedad entre hombres y mujeres a la hora de lograr sus objetivos, subrayando una visión holística, más que individual y/o autónoma, de la mujeres africanas.

Cornwall (2005) señala que existe un creciente corpus de trabajos que denuncian cómo las feministas occidentales han incomprendido profundamente la naturaleza del género y de las relaciones de género en África. Identificar las relaciones de género en África con un particular sistema jerárquico de relaciones de género entre hombres y mujeres, supone presumir que la dominación masculina es universal, y no tomar en cuenta otros tipos de diferencia que afectan a las mujeres africanas (como la generación, la familia o los grupos económicos a los que pertenecen), malinterpretando, de esta manera, su agencia (Cornwall, 2005: 4-5). Desde esta perspectiva podemos entender la solidaridad dentro de la familia que muchas africanas han 
antepuesto a la solidaridad entre mujeres (Ekejiuba, 2005; Oyewumi, 2001), como un aspecto clave para entender los feminismos africanos.

Si bien no existe una coincidencia de planteamientos entre las diferentes autoras africanas sobre el feminismo (Pérez Ruíz, 2011), Nnaemeka (2005) ha señalado que, a pesar de la pluralidad y la multiplicidad de perspectivas, es posible ver en todos ellos una tendencia hacia el poder compartido, la complementariedad, la acomodación, el compromiso, la negociación y la inclusividad. Siguiendo a Nnaemeka, la vida humana se ve de forma total (holística) en lugar de en una dicotómica y exclusiva perspectiva. Lo masculino no es «el otro» sino parte de la misma humanidad y cada género constituye una mitad crítica que hace lo humano como un todo. De esta manera las mujeres africanas están más inclinadas a alcanzar y trabajar con los hombres para conseguir sus objetivos (Nnaemeka, 2003).

En un esfuerzo de síntesis encomiable, Arndt clasifica tres corrientes feministas africanas que califica de reformista, transformativa y radical, donde ubica a las diferentes autoras africanas, en base a las reivindicaciones que aparecen en sus obras (Arndt, 2002). De su análisis se deduce la polifonía de las posturas africanas. Sin embargo, también aparecen dos cuestiones que se repiten en todas las autoras interpretadas por Arndt: la necesidad de ubicar el género en contexto, junto a la raza, la clase, la cultura, la economía, la casta, la etnia, la nación y otras consideraciones; y la necesidad de nociones de complementariedad y cooperación con los hombres, en la lucha por la transformación social. Podemos recoger algunos ejemplos de autoras africanas que defienden la complementariedad de hombres y mujeres dentro de sus posturas feministas a partir del análisis de Ardnt (2002) y de Pérez Ruíz (2011): Alice Walker; Clenora Hudson Weems; Molara Ogundipe-Leslie; Catherine Obianuju Acholonu; Obioma Nnaemeka; Oyeronke Oyewumi; Chikwenye Okonjo Ogunyemi; Mary E. Modupe Kolawole. También Zirion e Idarraga (2014) han recogido esta idea de reivindicar la igualdad desde lo comunitario por parte de los feminismos africanos, donde se subraya la colaboración de sus compañeros hombres como aliados, en la lucha por la igualdad.

Ogundipe-Leslie (2002) resume siete denominadores comunes del feminismo en el contexto africano, de entre los cuales vamos a destacar los siguientes:

1.- que el feminismo no necesita oponerse a los hombres. No se trata de una política de confrontación de los sexos; [...] 6.- que las condiciones de las mujeres en África necesitan ser contempladas en el contexto total de la producción y la reproducción de sus sociedades, incluyendo en este escenario también a los hombres y a los niños. De ahí que siempre haya habido un énfasis sobre la realización e independencia económica en el pensamiento feminista africano; 7.- que la ideología de las mujeres tiene que ser proyectada sobre el contexto de las luchas de raza y clase que atenazan al continente africano actualmente, es decir, en el contexto de la liberación de todo el continente. Generalmente, es esta actitud holística de las mujeres africanas con respecto al feminismo lo que a menudo las separa de sus hermanas en occidente (Ogundipe-Leslie, 2002: 78-79). 
En definitiva, sin perder de vista la línea teórica en la que enmarcábamos esta investigación, que subraya los procesos migratorios como factores de estrés que pueden desencadenar tensiones en algunos matrimonios (sobre todo alrededor de lo que se ha denominado maltrato económico y negligencia), es necesario retener esta actitud holística que han puesto de manifiesto los feminismos africanos y/o afrocéntricos, para comprender qué mecanismos operan a la hora de poner en marcha las estrategias híbridas que se recogen en los casos que mostraremos a continuación. La visión holística sobre las relaciones de género y la noción de complementariedad que defienden la mayoría de voces de mujeres africanas, puede ayudarnos a entender las líneas de acción y las diferentes estrategias que las mujeres migrantes africanas han puesto en marcha en contextos transnacionales para encarar los conflictos en la pareja. Veremos cómo incluyen a los hombres en sus estrategias, cómo usan las redes familiares/comunitarias, en muchas ocasiones transnacionales, a la vez que ponen en marcha procesos de hibridación que ponen en valor la experiencia de mujeres africanas reconocidas.

\section{Apuntes metodológicos}

El presente artículo se basa en el trabajo de campo realizado entre 2017 y 2018 en una Comarca rural de Aragón, a la que hemos llamado La Aldahuela de Osca. El acceso al campo fue posible gracias al trabajo desarrollado en una Administración pública local de esta zona geográfica. A partir de este trabajo surgió el contacto con las Asociaciones de mujeres inmigrantes y se pudo establecer relaciones de confianza con muchos/as inmigrantes de la zona.

La Aldahuela de Osca representa simbólicamente una Comarca de Aragón cuyas características demográficas y económicas resultan similares a otras zonas estudiadas en esta Comunidad Autónoma, donde las tareas de recolección frutícola y la agroindustria han ido dibujando un mapa migratorio donde, en un primer momento, los flujos procedentes de África subsahariana han despegado con la estacionalidad propia de las rutas temporeras de la recolección de la fruta, y, en un segundo momento, se han asentado a partir de las posibilidades que la agroindustria ha proporcionado a los inmigrantes subsaharianos en estas zonas (Ródenas, 2019; Pinos, 2001). A partir de este escenario, aparecieron las primeras familias transnacionales africanas, y las mujeres pudieron establecerse en la zona a partir de las primeras reagrupaciones familiares que realizaron sus maridos.

Específicamente, la investigación que presentamos consistió, en un primer momento, en un acercamiento a las Asociaciones de mujeres inmigrantes procedentes de Mali, Gambia y Senegal que se han creado en la zona, a través de grupos de discusión donde se identificaron los principales temas recurrentes que preocupan a las mujeres. En total se trabajó con 6 asociaciones de mujeres inmigrantes que llamaremos Mali1, Mali2, Gambia1, Senegal1, Plurinacional1 y Plurinacional-religiosa2. Se realizaron también entrevistas semiestructuradas, individuales y preliminares, a mujeres representantes de estas asociaciones africanas. A partir de los datos recabados con los grupos de discusión y con las entrevistas preliminares, se eligió a 5 mujeres para 
realizar las entrevistas en profundidad, atendiendo a los criterios que marcaron los resultados del propio desarrollo de la investigación: y es que, a partir de los grupos de discusión y de las entrevistas preliminares realizadas, se identificó a estas 5 mujeres que actuaban de referente para el resto de sus compatriotas a la hora de producirse tensiones, conflictos, problemas o incidentes en los matrimonios. Las entrevistas en profundidad a estas 5 mujeres referentes, se realizaron en espacios privados, generalmente en sus casas particulares, donde las mujeres se sentían más cómodas, controlaban los espacios y los tiempos que dedicábamos, y tenían suficiente intimidad y confianza para hablar de temas tan delicados como son "cosas de mujeres», que, como veremos en los extractos etnográficos reproducidos en los apartados posteriores, se refiere a «hablar de secretos de mujeres, cosas de mujeres, hijos, sexualidad...» (Diario de campo, Aminata, 2018).

En cuanto a las 5 mujeres elegidas, sus principales características se recogen en el siguiente cuadro:

\begin{tabular}{|lllll|}
\hline PSEUDÓNIMOS & ORIGEN & $\begin{array}{l}\text { EDAD } \\
(\mathrm{años})\end{array}$ & RUTA MIGRATORIA & SITUACIÓN FAMILIAR \\
\hline Mariam & Mali & 40 & Reagrupada por su marido & $\begin{array}{l}\text { Divorciada primero y actualmen- } \\
\text { te viuda. 4 hijos/as }\end{array}$ \\
\hline Aminata & Mali & 41 & Reagrupada por su marido & $\begin{array}{l}\text { Divorciada, se ha vuelto a casar. } \\
\text { Una hija de cada matrimonio }\end{array}$ \\
\hline Isatu & Gambia & 53 & Reagrupada por su marido & $\begin{array}{l}\text { Casada como segunda mujer, } \\
\text { actualmente única.4 hijos/as pro- } \\
\text { pios y 2 de la primera mujer }\end{array}$ \\
\hline Bintu & Gambia & 40 & Reagrupada por su marido & $\begin{array}{l}\text { Primera mujer. Coesposa vive en } \\
\text { Gambia. 7 hijos/as }\end{array}$ \\
\hline Awa & Senegal & 50 & $\begin{array}{l}\text { Viaja sola a Francia donde } \\
\text { está su hermano. De ahí a } \\
\text { España }\end{array}$ & $\begin{array}{l}\text { Mujer única casada en destino } \\
\text { (España). 2 hijas }\end{array}$ \\
\hline
\end{tabular}

A continuación pasamos a reproducir algunos extractos etnográficos de las narraciones producidas durante las entrevistas, con una intención analítica y expresiva (Bertaux, 1989), esto es, con una intención de comparar casos para interpretar la realidad, a la vez que se ilustran algunas de las explicaciones teóricas que se pretenden poner a prueba en el presente texto.

\section{Narraciones etnográficas: conflictos y estrategias de resolución en familias transnacionales africanas}

Como veíamos en apartados anteriores, existen diversos estudios que ponen de manifiesto las opciones que en contexto migratorio tienen hombres y mujeres africanas dentro de la comunidad a la hora de solucionar agravios y conflictos (Akinsulure-Smith et al., 2013; Ogunsiji et al., 2011; Pan et al., 2006; Sokoloff, 2008; Ting, 2010; Ting y Panchanadeswaran, 2009). 
En este sentido, es necesario tomar en consideración que, en un contexto migratorio transnacional, cambian roles y se adaptan los comportamientos, de modo que este procedimiento de resolución de conflictos que se ha esbozado en apartados anteriores, puede llegar a sufrir algunos cambios y modificaciones. Durante el trabajo realizado en esta investigación se ha podido constatar cómo los procesos de resolución de conflictos dentro de las familias transnacionales que provienen de África Occidental, repiten unos patrones muy particulares. Veremos a continuación varias vías disponibles para la resolución de conflictos maritales: en el momento en que las mujeres africanas se ven inmersas en conflictos dentro de sus matrimonios tienen la posibilidad de acudir a los miembros de su familia extensa transnacional para que intercedan ante la familia del marido; o pueden acudir a mujeres de referencia dentro de sus comunidades que pueden escuchar, aconsejar y, en algunos casos, movilizar capital social ${ }^{3}$, para apoyar y proteger a sus confidentes; mientras que en otros casos la comunidad puede presionar en contra de los intereses de la mujer. De esta manera el contexto transnacional influye en cómo se adaptan las estrategias. Para ejemplificar esta variedad de procesos en la resolución de conflictos, vamos a exponer casos diferentes procedentes de Mali, Gambia y Senegal.

\section{$\underline{\text { MALI }}$}

Mariam nació en 1979 en Mali. Es presidenta de una de las asociaciones de mujeres más activas del municipio. Llegó hace 18 años, actualmente viuda, antes divorciada, madre de 4 niños, ahora mayores de edad. Ella habla de pedir ayuda desde la asociación:

Para las mujeres, para poder ponerse frente a los hombres [...] Antes los hombres africanos no querían que sus mujeres trabajaran, tú no has venido aquí a trabajar, pero ahora sí, hay muchos niños y ellos tienen poco trabajo. Y a algunos les da igual que tú aquí pases hambre con tus hijos, ellos quieren mandar allí, a su familia, y tu familia da igual... sobre todo cuando cogen otra [...] ahora que las mujeres trabajan, sus maridos les piden que paguen gastos, facturas... a veces les quitan el dinero.

Mariam explica sus propias vivencias: «yo puedo hablar de mí... estoy viviendo aquí mucho tiempo, tengo que respetar a mi marido, si él tiene problemas, tengo que ayudar, si yo tengo problemas, él tiene que ayudar». Cuenta que traer a su mujer aquí: «es como tu esclava, tiene que dar el dinero a él, yo nunca hice esto, abrí mi cuenta en el banco cuando empecé a trabajar, y me decía, tú has cambiado, como los blancos, él dice, te voy a mandar a África, porque yo no lo respeto». Cuando Mariam decidió divorciarse, su familia en África la apoyó:

A veces mi madre me manda dinero por Money Gram, porque es una vergüenza mandarme dinero, dar a alguien para que traiga... mi padre tiene un

3 El capital social fue definido por Bourdieu (1980) como : «l'ensemble des ressources actuelles ou potentielles qui sont liées à la possession d'un réseau durable de relations plus o moins institusionnalisées d'interconnaissance et d'interreconnaissance; ou en d'autres termes, à lsappartenance à un groupe» (Bourdieu, 1980: 2, cursiva en el original). 
poco de dinero, es comerciante, tiene pisos alquilados, es mi familia que me ayuda [...] Mi familia a principio me decía aguanta, pero vieron que era un mentiroso, que se portaba muy mal, tenía envidia de mí, yo empecé a salir adelante y a él no le gustaba que me fueran bien las cosas. Aquí hay muchas mujeres que están así, que sus maridos no les dan dinero, que no les dejan trabajar, es muy difícil, lo pasan muy mal, pero algunas tienen miedo, o no saben salir adelante y aguantan.

Aminata nació en Costa de Marfil, pero su familia es de Mali y ha vivido en Mali desde los 2 años. Se casó con 14 años, y llegó reagrupada en 2003, con 25 años, aunque actualmente está divorciada de él. Tiene una hija mayor de su primer marido y está embarazada de su segundo marido, con quien se casó aquí tras su divorcio.

Cuando surgen problemas matrimoniales, Aminata pone el acento en el «valor» de la mujer:

Todo depende de si tienen valor, no todas tienen, porque te van a hacer sufrir... Te dicen, yo te traigo, te doy comida, te doy todo, y me puedo casar con otra porque lo dice el Corán. Hay que tener valor para afrontar y contar. Tú dices a los hombres que vas a hablar de secretos de mujeres, cosas de mujeres, hijos, sexualidad... esto tienes que decir a los hombres, porque si no, no te dejan hablar con mujeres. Hablar con mujeres divorciadas... Están mal vistas, porque te van a decir que te separes.

Aminata me presenta a varias mujeres que han ido a verla. Me cuenta el caso de Fatu. El marido lleva más de diez años en La Aldahuela de Osca, es querido y respetado. Ella (Fatu) estaba en Zaragoza, ahora hace casi un año que vino a La Aldahuela de Osca. Tuvo problemas con su marido, acudió a Servicios Sociales y puso una denuncia de violencia de género, que luego retiró. Fatu no encuentra a nadie de la comunidad africana que quiera cuidar a sus tres hijos. Aminata explica: «tiene boca, habla demasiado... Es una nueva persona pero su nombre está en boca de todos. Es malagradecida, se mete en todo, comenta demasiado... Yo conozco a su marido y no creo lo que ella dice». Al cabo de los meses, Fatu se mudará a otro municipio, donde tiene una amiga que puede apoyarla.

También me presenta a Fanta: Fanta llegó reagrupada por su marido:

Empecé a trabajar y empezamos a pelear, quería que le diera mi dinero, él me había traído, y me daba 5 euros, 10 euros... yo no quería. Hablé con su familia y me dijeron que él no va a coger mi dinero más. Pero sus padres se pusieron enfermos y fui a Mali a cuidarlos, me quitó el pasaporte, el NIE y me dijo que nunca más volvería a España.

\section{GAMBIA}

Isatu llegó reagrupada por su marido a Cataluña en 1993, tenía entonces 27 años. Después de vivir dos años allí, se mudaron a La Aldahuela de Osca, donde han residido hasta la actualidad. Ha sido segunda mujer de su marido, si bien ahora es la única que mantiene. Tiene 4 hijos en España, ya casados y emancipados, y 
además también ha criado en destino a los otros dos hijos de su marido con su primera mujer. Isatu participa en diferentes asociaciones y ha liderado varias de ellas.

Cuando hay problemas la mujer habla con un amigo de su marido, para que hable con su marido. A veces se enfadan más porque la mujer ha contado, y es peor. Mira, una chica viene a hablar conmigo el otro día, me cuenta, mi marido llama a él, quiero hablar contigo, y su marido no quiere, vale. Cuelga y le dice a ella, puedes quedarte aquí. Si mi marido habla, no llega a esto. Y ella no es mi familia, pero da igual, lo que le ha pasado a ella puede pasarle a mi hija [...] Otro día vino otra mujer, Haja. Ella lleva años aquí, con cuatro hijos, ya es abuela de la mayor, y su marido... Ella duerme en el suelo, hace meses que no sexo, él tiene allí dos mujeres más y habla por teléfono con ellas delante de Haja, a risas... Tiene allí dos hijos de ella que él no quiere traer, si los trajera la podrían ayudar a ella, pero ella no quiere hablar... no cuenta.

Unas semanas después Haja, desesperada, acudirá a la Guardia Civil a denunciar a su marido porque, dice, manda el dinero a África y a ella no le da para comer ni para sus hijos. La Guardia Civil le dirá que debe poner una denuncia de violencia de género. Haja no la pondrá.

El marido de Bintu la reagrupó con 19 años, en 1998, llegó directamente a La Aldahuela de Osca. Cuenta que, por entonces, sólo había 2 o 3 mujeres gambianas en el pueblo. Ha tenido 7 hijos, y su marido tiene además otra mujer en Gambia, con otros 3 hijos. Bintu explica que ella no ha tenido nunca problemas con su marido.

Bintu narra:

Las mujeres se juntan para hablar. Si hay un problema, la mujer se lo cuenta a su familia que tenga aquí. Si aquí no hay familia va a una mujer con experiencia. Se habla con el padre de la chica en Gambia, y ellos miran si el matrimonio se separa o no. Se juntan los mayores para hablar. Si el marido no hace caso, se habla otra vez con el padre de él, y se pueden separar. Si el marido quiere a esa mujer, tiene que ir a hablar con el padre de ella otra vez. Si la mujer no quiere, pues nada [...] El problema es que algunas no van a contar lo que les pasa, porque hay hijos, o por vergüenza, para que todo el mundo no cuente mi problema, porque si me cuenta a mí, yo no voy a callar. [...] Hay que aguantar, porque hay amor, él quiere verla, se preocupa de que los demás hombres la miren, pero hay cosas que no se pueden aguantar. Se puede perdonar una discusión, un enfado, pero si sigue, hay que dejarlo.

\section{SENEGAL}

Awa tiene 50 años y llegó a Francia hace más de 15 (donde estaba su hermano) desde Senegal, con un visado que caducó. Después de dos años en Francia, decidió venir a España, le habían dicho que aquí era más fácil regular su situación administrativa. Su madre llamó a alguien que conociera en España... alguien que se convertiría, con el tiempo, en su futuro marido. Tiene dos hijas con él y es mujer única. También lidera una de las Asociaciones de mujeres del lugar. 
Hablamos del asesinato de una senegalesa en un municipio del entorno rural donde vive, a manos de su marido también senegalés:

Se veía venir... Todos sabían lo que pasaba, todos los de La Aldahuela de Osca eran familia del chico y todos hablaban con ella para que le perdone, y mira lo que ha pasado. Ahora todos callan. La culpa es de ellos. Son familia y aconsejaban aguantar... ya no hay nada que aguantar... [...] En Senegal ahora también pasa, también las están matando, ya no hay que aguantar, los tiempos han cambiado.

\section{Análisis afrocéntricos de la violencia de género y agencia cultural híbrida en contexto migratorio: el caso de «una mujer con experiencia».}

En los ejemplos de maltrato económico y/o negligencia que hemos podido rescatar de las narraciones producidas por las mujeres entrevistadas, se ha puesto de manifiesto el tipo de tensiones que afectan a estos matrimonios y se han subrayado los mecanismos que se utilizan para proteger a la mujer que está siendo afectada por estos tipos de maltrato.

Resulta necesario destacar que los ejemplos mostrados de conflictos se pueden interpretar desde una perspectiva de relaciones de género dentro de los matrimonios africanos, donde los roles asignados a cada cónyuge delimitan las tareas y responsabilidades dentro de la familia trasnacional (Ródenas, 2014). De esta manera, sin perder de vista la posición desigual que las mujeres ocupan en la estructura social, podemos destacar que los problemas surgen cuando el marido no satisface sus funciones ni su responsabilidad derivada de sus roles tradicionales, esto es, no cubre económicamente lo necesario en la familia, mientras que la mujer sí cumple con sus roles haciendo de cuidadora, educadora, manteniendo la casa, gestionando las tareas domésticas, etc. En consecuencia la esposa acude al mercado laboral a trabajar, o demanda hacerlo sin que el marido se lo permita, o él intenta imponer su acceso al salario ganado por ella. Es en estas situaciones cuando las tensiones se disparan, y acudimos a conflictos que siguen los patrones arriba descritos.

En cuanto a los ejemplos de resolución de conflictos ilustrados, vemos principalmente dos procedimientos utilizados: usar las redes de la familia extensa trasnacional, o, en otras ocasiones, movilizar el capital social y las redes familiares en destino, a través de la comunidad. Ambas vías nos han permitido poner de manifiesto el papel central en contexto migratorio de «una mujer con experiencia» capaz de movilizar los respectivos linajes de los cónyuges afectados a través de sus redes, pero también a través de las redes desarrolladas por su marido. Y es aquí donde podemos ver cómo la complementariedad y el comunitarismo subrayado por diferentes voces de feministas africanas entran en juego, a la vez que se ponen en funcionamiento procesos de hibridez para reinventar en contexto migratorio prácticas tradicionales. En el momento en el que surgen los problemas en el matrimonio, y en ausencia de familia que las proteja en destino, las esposas implicadas acuden a estas mujeres con experiencia, quienes ponen en marcha los mecanismos de resolución, sopesando qué redes movilizar y de qué manera: cuando la posibilidad de contactar con la familia transnacional se diluye, pueden apoyar y aconsejar a la 
esposa agredida, o movilizar el capital social de los hombres, o, en otras ocasiones, defender al agresor y presionar para que la mujer «aguante». En este último caso, ni las redes extensas ni la comunidad en destino sirven para proteger a la mujer que denuncia el maltrato, sino, todo lo contrario, han aislado y presionado a la denunciante para «aguantar» y mantener el matrimonio, en ocasiones teniendo que mudarse a municipios vecinos, o teniendo otros finales mucho más trágicos.

En cualquiera de estas vías posibles, descritas en el presente trabajo, ha quedado patente una idea latente que es necesario contextualizar: la idea de «aguantar». Mantener el matrimonio solucionando los conflictos que puedan surgir, es una prioridad tanto para la familia de ambos cónyuges, como para la mujer protagonista de la historia. El divorcio sólo es una opción cuando el resto de estrategias no funcionan. En palabras de Dial (2008):

L'institution matrimoniale continue de décider fortement des modes de la valorisation et du déclassement social. À Dakar le mariage reste valorisé et valorisant pour les femmes. Le cadre de vie nécessaire à leur épanouissement continue d'être le foyer conjugal. Toutes les femmes y aspirent. Aussi important que puisse être leur niveau d'instruction ou la fonction occupée dans un poste de travail, elles reconnaissent le besoin d'un référent nouveau qui ne doit plus être, passé la puberté, le père ou l'oncle, mais le mari. Ce besoin s'exprime sous une réelle pression familiale et sociale (Dial, 2008: 97).

De esta manera, proteger a la mujer que está inmersa en estos conflictos implica presionar al marido para que cambie su comportamiento, y si persiste, facilitar la separación. Por estos motivos prevenir e intervenir en los casos de violencia de género que se producen en familias transnacionales africanas en los contextos de destino que hemos analizado, implica primero, detectar posibles situaciones de maltrato económico y/o negligencia que no siempre se conceptualizan ni se pueden acreditar como propiamente parte de la «violencia de género», y, segundo, trabajar con programas de intervención culturalmente apropiados e implementados en el nivel comunitario (Akinsulure-Smith et al., 2013; Pan et al., 2006). En conclusión, dar formación, recursos y herramientas a aquellos y aquellas (llamadas en nuestros casos «mujeres con experiencia») que tienen la capacidad de movilizar redes trasnacionales y/o comunitarias para proteger a las mujeres que se encuentran en situaciones adversas, pero teniendo cuidado de no señalarlas en exceso, para evitar que sean estigmatizadas por su propia comunidad.

En definitiva, las formas de resolución de conflictos dentro de los matrimonios africanos debería hacernos reflexionar sobre cómo se construyen las relaciones de género histórica, social y culturalmente por diferentes grupos, ya que la solidaridad comunitaria, la familia extensa transnacional y la complementariedad entre hombres/mujeres juegan un papel muy importante a la hora de proteger o no a la mujer denunciante en los casos de violencia de género que estamos analizando. Pero no sólo es necesario incorporar otros análisis, como el conocimiento que aportan los feminismos africanos/ afrocéntricos, sino que además deberíamos dar cuenta de los procesos de hibridación que surgen en los procesos migratorios trans- 
nacionales. La relevancia de «una mujer con experiencia» que emerge de un procedimiento tradicional como es la resolución de conflictos matrimoniales a través de la intervención de la familia extensa, no deja de ser un ejemplo de adaptación al nuevo contexto migratorio, y denota la capacidad de adaptación, cambio, hibridación y agencia que se posee desde las culturas africanas contemporáneas. Y es justo en ese contexto de agencia cultural híbrida, donde hemos pretendido ubicar la emergencia de «una mujer con experiencia» en la presente investigación.

\section{Referencias bibliográficas}

Abu-Lughod, Lila (2002). «Do muslim women really need saving? Anthropological reflections on cultural relativism and its others» en American Anthropologist, $\mathrm{N}^{\circ} 104$ (3), pp. 783-790.

Akinsulure-Smith, Adeyinka et al. (2013). «Intimate Partner Violence among West African Immigrants» en J Aggress Maltreat Trauma, No22 (1), pp. 109-129.

Amadiume, Ifi (2018). Hijas que son varones y esposos que son mujeres. Género y sexo en una sociedad africana, Barcelona: Edicions Bellaterra.

Amos, Valerie y Pratibha PARMar (1984). «Challenging imperial feminism» en $F e$ minist Review, No17, pp. 3-19.

ApPerT, Catherine (2016). «On Hybridity in African Popular Music: The Case of Senegalese Hip Hop» en Ethnomusicology, No60 (2), pp. 279-299.

ARNDT, Susan (2002). The dynamics of African Feminism. Defining and classifying African Feminist Literatures, Trenton, NJ; Asmara, Eritrea: Africa World Press, Inc.

Azoulay, Muriel y Catherine Quiminal (2002). «Reconstruction des rapports de genre en situation migratoire. Femme 'réveillées', hommes menaces en milieu soninké» en VEI Enjeux, Nº128, pp. 87-101.

BaKARE-Yusuf, Bibi (2011). «Los Yoruba no hacen género: Una revisión crítica de 'La invención de la mujer: Haciendo un sentido africano de los discursos occidentales de género', de Oyewumi Oyeronke» en Africaneando. Revista de actualidad y experiencias, $\mathrm{N}^{\circ} 5$, pp. 25-53.

BERTAUx, Daniel (1989). «Los relatos de vida en el análisis social» en Historia y Fuente Oral, No1, pp. 87-96.

Bнава, Homi (2002). El lugar de la cultura, Buenos Aires: Ediciones Manantial (Original 1994).

BouRdieu, Pierre (1980). «Le capital social» en Actes de la recherche en sciences sociales, No31, pp. 2-3.

Bowman, Cynthia (2003). «Theories of Domestic Violence in the African Context» en Cornell Law Faculty Publications. Paper 131.

Bryceson, Deborah y Ulla Vuorela (2002). «Transnational families in the twentyfirst century», en Bryceson, Deborah y Ulla Vuorela (eds.) (2012), The transnational family: new European frontiers and global networks. Oxford; New York: Berg Publishers, pp.15-42. 
CARby, Hazel (2012). «Mujeres blancas, jescuchad! El feminismo negro y los límites de la hermandad femenina», en Jabardo Velasco, Mercedes (ed.) (2012), Feminismos negros. Una antología. Madrid: Traficantes de Sueños, pp.209-243

Cornwall, Andrea (2005). "Introduction: Perspectives on gender in Africa», en Cornwall Andrea (ed.) (2005), Readings in gender in Africa. Bloomington: Indiana University Press; Oxford: James Curray, pp. 1-19.

Crenshaw, Kimberlé Williams (2012). «Cartografiando los márgenes. Interseccionalidad, políticas identitarias, y violencia contra las mujeres» en PLATERo MÉNDEZ, Raquel Lucas (coord.) (2012). Intersecciones: cuerpos y sexualidades en la encrucijada, Barcelona: Bellaterra, pp. 87-122.

Damonti, Paola (2019). «Exclusión social como factor de riesgo de violencia de género en la pareja» en Papers. Revista de Sociología, No104 (3), pp. 485-523.

Dial, Fatou Binetou (2008) Mariage et divorce à Dakar: itinéraires féminins, Dakar: Karthala Editions.

EKejiuba, Felicia (2005). «Down to fundamentals: women-centred hearth-holds in rural West Africa», en CoRnwall, Andrea (ed.) (2005) Readings in gender in Africa. Bloomington: Indiana University Press; Oxford: James Curray, pp. 41-46.

García CANCLINI, Néstor (1990). Culturas Híbridas. Estrategias para entrar y salir de la modernidad, Mexico: Editorial Grijalbo.

(1999). Interculturalidad e Hibridación Latino. Mexico: Universidad Autónoma Metropolitana Iztapalapa. Disponible en: https://www.google.com/ url?sa $=\mathrm{t} \& \mathrm{rct}=\mathrm{j} \& \mathrm{q}=\&$ esrc $=\mathrm{s} \&$ source $=$ web \&cd $=\& \mathrm{cad}=\mathrm{rja} \& u a c t=8 \& \mathrm{ved}=2 \mathrm{ahUK}$ Ewjkxo_G-oHuAhWQRRUIHXDiByIQFjAAegQIBRAC\&url=http $\% 3 A \% 2 F \% 2$ Fred.pucp.edu.pe\%2Fwp-content $\% 2$ Fuploads $\% 2 F b i b l i o t e c a \% 2 F n e s t o r g a r c i a c a$ ncliniinterculturalidadehibridacionlatino.pdf\&usg=AOvVaw2iW1guP6bvo3uH7A24RY6 (Consultado: 28 de Diciembre de 2020).

INIESTA, FERRAN (2010) El pensamiento tradicional africano. Regreso al planeta negro, Madrid: Los Libros de la Catarata, Casa África.

Jabardo Velasco, Mercedes (2005). «Migraciones y género. Cuando el continente africano se hace pequeño» en Revista Española de Desarrollo y Cooperación, N¹6, pp. 81-98.

(2012). Feminismos negros. Una antología. Madrid: Traficantes de Sueños.

KonAté, Famagan-Oulé (2010). «La migration féminine dans la ville de Kayes au Mali» en Hommes E Migrations, Nº1286-1287, pp. 62-73.

Mohanty, Chandra Talpade (2008). «Bajo los ojos de Occidente: academia feminista y discursos coloniales», en SuÁrez NAvaz, Liliana y Rosalva Hernández Castillo (eds.) (2008), Descolonizando el feminismo. Madrid: Cátedra, pp. 117163.

NnaemeKa, Obioma (2003). «Nego-Feminism: Theorizing, Practicing, and Pruning Africa's Way» en Signs: Journal of Women in Culture and Society, No29 (2), pp. 357-385.

(2005). «Mapping african feminism», en Cornwall, Andrea (ed.) (2005) Readings in gender in Africa. Bloomington: Indiana University Press; Oxford: James Curray, pp. 31-41. 
Ogundipe-Leslie, Molara (2002). «Stiwanismo en un contexto africano» en Arenal, $\mathrm{N}^{\circ} 9(1)$, pp. 59-92.

OgunsISJI, Olayide et al. (2011). «Suffering and smiling: West African Immigrant Women's Experience of Intimate Partner Violence» en Journal of Clinical Nursing, N21, pp. 1659-1665.

Oyewumi, Oyeronke (2001). «Ties that (un)bind: feminism, sisterhood and other foreign relations» en JENdA: A journal of cultura and african women studies, Issue 1.

PAN, Amy et al. (2006). "Understanding the Role of Culture in Domestic Violence: The Ahimsa Project for Safe Families» en Journal of Immigrant and Minority Health, No8 (1), pp. 35-43.

PÉREz Ruíz, Bibian (2011) Otra manera de sentir: feminismos negros, género y estudios literarios en el África Subsahariana, XII Edición del Premio Elisa Pérez Vera (UNED).

Pinos Quílez, Manuel (2001) «Immigrants i temporers estrangers al Baix Cinca 'Construint junts l'únic futur possible'» en Cinga. Anuari de l'Institut d'Estudis del Baix Cinca.

RaJ, Anita y Jay Silverman (2002). «Violence Against Immigrant Women» en Violence Against Women, No8 (3), pp. 367-398.

RED2RED, Grupo (2011). El Estado de la Cuestión en el Estudio de la Violencia de Género. Madrid: Ministerio de Sanidad, Servicios Sociales e Igualdad.

Rey Anacona, César (2013). «Prevalencia y tipos de maltrato en el noviazgo en adolescentes y adultos jóvenes» en Terapia Psicológica, No31 (2), pp. 143-154.

RódenAs CEREzo, Beatriz (2014). Redes, matrimonio y agencia. Mujeres transnacionales entre Valencia y Senegal, Tesis de Doctorado, Elche: Universidad Miguel Hernández.

(2019). «'Como pajaritos...' Fruticultura, migración y género en los enclaves rurales del río Cinca» en Temas de Antropología Aragonesa, N²5, pp. 249-278.

Rodríguez-García, Dan (2013). «La abominación de lo híbrido: la mixofobia como política de Estado» en Glocalism: Journal of Culture, Politics and Innovation, $\mathrm{N}^{\circ} 1$.

RosAldo, Michelle Zimbalist (1979). «Mujer, cultura y sociedad: una visión teórica» en Harris, Olivia y Kate Young (eds.) (1979). Antropología y feminismo, Barcelona: Anagrama, pp. 153-180 (Original 1974).

(1980). "The use and abuse of Anthropology: reflections on feminismand cross-cultural understanding» en Signs, No5 (3), pp. 389-417.

SoKoloff, Natalie (2008). «The Intersectional Paradigm and Alternative Visions to Stopping Domestic Violence: What Poor Women, Women of Color, and Immigrant Women are Teaching us About Violence in the Family» en International Journal of Sociology of the Family, No34 (2), pp. 153-185.

SuÁrez Navaz, Liliana y Rosalva Hernández CAstillo (eds.) (2008). Descolonizando el feminismo. Madrid: Cátedra.

TING, Laura (2010). «Out of Africa: Coping Strategies of African Immigrant Women Survivors of Intimate Partner Violence» en Health Care for Women International, No31 (4), pp. 345-364.

Ting, Laura y Subadra Panchanadeswaran (2009). «Barriers to Help-Seeking Among Immigrant African Women Survivors of Partner Abuse: Listening to 
Women's Own Voices» en Journal of Aggression, Maltreatment \& Trauma, $\mathrm{N}^{\circ} 18$ (8), pp. 817-838.

VV.AA (2004). Otras Inapropiables. Feminismos desde las Fronteras. Madrid: Traficantes de Sueños.

Zirion Landaluze, Iker y Leire IdARRAga Espel (2014). «Los feminismos africanos. Las mujeres africanas 'en sus propios términos'» en Relaciones Internacionales, $\mathrm{N}^{\circ} 27$, pp. 35-54.

Recibido el 22 de mayo de 2020

Aceptado el 5 de enero de 2021

BIBLID [1132-8231 (2021): 195-215] 